\title{
The distribution of pollutants in the ground layer of the atmosphere in the presence of forest plantations
}

\author{
Alexander E. Chistyakov ${ }^{1, *}$, Alla $V$. Nikitina $^{2}$ and Elena A. Protsenko ${ }^{3}$ \\ ${ }^{1}$ Don State Technical University, Rostov-on-Don, Russia \\ ${ }^{2}$ Southern Federal University, Taganrog, Russia \\ ${ }^{3}$ Taganrog Institute named after A.P. Chekhov Rostov State Economic University (RINH), Taganrog, \\ Russia
}

\begin{abstract}
The aim of the work is to study the influence of forest plantations on the distribution of pollutants in the ground layer of the atmosphere. The model that takes into account a variety of factors: the presence of forest plantations, the variability of pressure, density and temperature, the presence of a multicomponent impurity, etc., was proposed for the numerical modeling of the process of transferring air pollutants to air. The scheme obtained as a result of a linear combination of the central difference scheme and the «CABARET» scheme was constructed to approximate the convection operator in this paper. An analysis of the results of numerical experiments allows to conclude that the distribution of pollutants in a multicomponent air environment is most significantly affected by the density of vegetation, and insignificantly influenced by the width of the forest plantations area.
\end{abstract}

\section{Introduction}

The growing anthropogenic load adversely affects to the state of the ground layer of the atmosphere. In conditions of modern reality, it is impossible to avoid receipt of pollutants into the air ecosystem. At present, a wide range of theoretical studies on atmospheric air pollution based directly on the solution of differential equations is being conducted. Problems of this type are quite complex from a practical point of view and very significant. Mathematical formulation is not universal enough in works devoted to these problems.

The transfer of impurities in the atmosphere is significantly influenced by the wind regime and temperature stratification of the ground layer of the atmosphere, as well as meteorological conditions. Analysis and forecast of pollutants dispersion in the atmosphere is necessary for the rational allocation of residential areas and industrial enterprises whose production waste affects health and comfortable living conditions. The problems devoted to the analysis of atmospheric air require a large amount of initial data and complex

\footnotetext{
* Corresponding author: cheese 05@mail.ru
} 
mathematical methods of solution. In connection with the growth of anthropogenic impact on ecosystems, the actual problem of research is the improvement of mathematical models intended for assessing the state of the air environment. The mathematical modeling is effective way to solve this problem. It allows to numerically calculate the propagation fields of pollutants for various widths and densities of forest massifs in the ground layer of the atmosphere. The use of the interrelated mathematical models complex of the variability of the atmosphere gas and aerosol composition makes it possible to predict possible prospects for the development of the ecosystem and the consequences for the development of building projects, gardening, etc.

Significant factors in the development of aerial ecosystems are the aerodynamic processes that determine the transfer of pollutants and mixing of the air.

\section{Mathematical model}

The mathematical model of the impurity propagation process, presented in [1, 2], gives a theoretical description of this process, takes into account a number of physical factors that influence the transfer of the impurity, and allows one to predict its course and consequences.

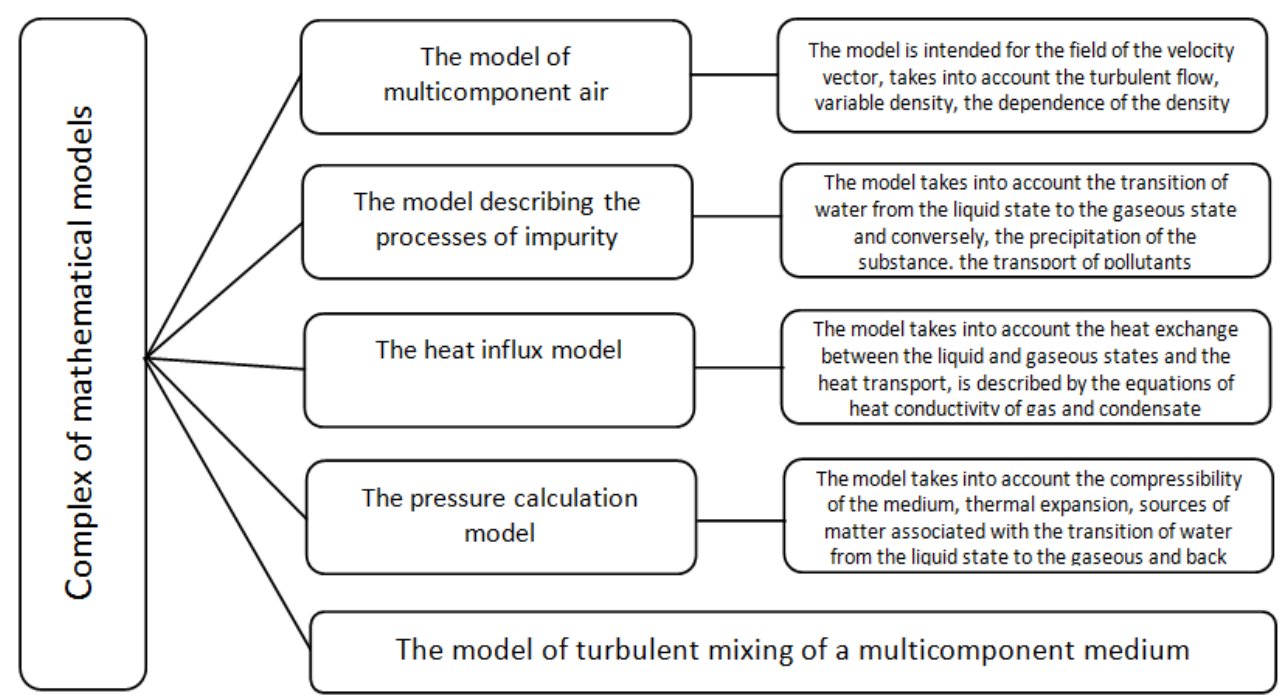

Fig. 1. Elements of the interrelated models complex.

Impurity scattering takes into account turbulence, heat and humidity propagation, the presence of forest massifs in the presented model. These additional conditions form a system and begin to act as a single set. Developed models also take into account the presence of forest plantations. Using the "CABARET" schemes allowed the authors of this work to develop a mathematical model that has the property of stability for a wider class of input parameters [3].

A set of models used to describe the motion of the air environment and the impurities propagation in it are presented in Figure 1. The initial equations of the model are shown in Table 1. 
Table 1. Initial model equations

\begin{tabular}{|c|c|}
\hline $\begin{array}{c}\text { The equation of motion } \\
\text { (the Navier-Stokes equation) }\end{array}$ & $\frac{d v_{j}}{d t}=-\frac{1}{\rho} \frac{\partial P}{\partial x_{j}}+\operatorname{div}\left(\mu \operatorname{grad}\left(v_{j}\right)\right)-g_{i}$ \\
\hline $\begin{array}{c}\text { The equation of continuity (transport } \\
\text { of matter) }\end{array}$ & $\frac{\partial \rho}{\partial t}+\operatorname{div}(\rho \vec{v})=\operatorname{div}(\mu \operatorname{grad}(\rho))+I_{\rho}$ \\
\hline $\begin{array}{c}\text { The equation of state (an analog of the } \\
\text { Mendeleev-Clapeyron equation) }\end{array}$ & $P=\sum_{i} \rho_{i} R T / M_{i}$ \\
\hline The equation of impurity transport & $\frac{d \varphi_{i}}{d t}=\operatorname{div}\left(\mu \operatorname{grad}\left(\varphi_{i}\right)\right)+I_{\varphi}$ \\
\hline The equation of heat influx & $\frac{d Q}{d t}=\operatorname{div}(\mu \operatorname{grad}(Q))+\operatorname{div}(\lambda \operatorname{grad}(T))+I_{Q}$ \\
\hline The equation of the turbulence model & $v_{S G S}=\left(C_{S} \Delta\right)^{2} S$ \\
\hline
\end{tabular}

In the Table $1 \varphi_{i}$ is the volume fractions of the $i$-th phases: air, water in the gaseous state, gas at the source, water in the liquid state, soot, $v_{j}$ the projection of the air velocity vector on the axis $O x_{j}, j=1,2,3 ; \lambda$ the coefficient of thermal conductivity, $R$ the universal gas constant, $T$ the gas phase temperature, $Q$ the thermal energy, $\mu$ the coefficient of turbulent diffusion, $\rho$ the density, $M$ the molar mass, $I$ the source function, $P$ the pressure [4].

\section{Difference scheme for the two-dimensional convection- diffusion equation}

Let consider the two-dimensional convection-diffusion equation

$$
\frac{\partial q}{\partial t}+u \frac{\partial q}{\partial x}+v \frac{\partial q}{\partial y}=\mu \frac{\partial^{2} q}{\partial x^{2}}+\mu \frac{\partial^{2} q}{\partial y^{2}},
$$

where $t \in[0, T], x \in\left[0, L_{x}\right], y \in\left[0, L_{y}\right] q(0, x, y)=q^{0}(x, y), q(t, 0, y)=q\left(t, L_{x}, y\right)=0$, $q(t, x, 0)=q\left(t, x, L_{y}\right)=0$.

Let cover the domain of definition by a uniform computational grid $\omega=\omega_{\tau} \times \bar{\omega}_{x} \times \bar{\omega}_{y}$, where $\bar{\omega}_{x}=\left\{x_{i} \mid x_{i}=i h_{x}, i=0,1, \ldots, N, N h_{x}=L_{x}\right\}, \bar{\omega}_{y}=\left\{y_{i} \mid y_{i}=i h_{y}, i=0,1, \ldots, N, N h_{y}=L_{y}\right\}$, $\omega_{\tau}=\left\{t_{j} \mid j=0,1, \ldots\right\}, \tau=t_{n+1}-t_{n}=$ const .

The scheme (2) for equation (3) will have the form:

$$
\begin{gathered}
\frac{q_{i, j}^{n+1 / 2}-q_{i, j}^{n}}{\tau}+\psi_{x L} \frac{q_{i-1, j}^{n-1 / 2}-q_{i-1, j}^{n-1}}{2 \tau}+\psi_{x R} \frac{q_{i+1, j}^{n-1 / 2}-q_{i+1, j}^{n-1}}{2 \tau}+ \\
+u \frac{q_{i+1, j}^{n}-q_{i-1, j}^{n}}{4 h_{x}}+\psi_{x L} u \frac{q_{i, j}^{n}-q_{i-1, j}^{n}}{h_{x}}+\psi_{x R} u \frac{q_{i+1, j}^{n}-q_{i, j}^{n}}{h_{x}}=\frac{3}{2} \mu \frac{q_{i+1, j}^{n}-2 q_{i, j}^{n}+q_{i-1, j}^{n}}{h_{x}^{2}} \\
\frac{q_{i, j}^{n+1}-q_{i, j}^{n+1 / 2}}{\tau}+\psi_{y L} \frac{q_{i, j-1}^{n}-q_{i, j-1}^{n-1 / 2}}{2 \tau}+\psi_{y R} \frac{q_{i, j+1}^{n}-q_{i, j+1}^{n-1 / 2}}{2 \tau}+v \frac{q_{i, j+1}^{n+1 / 2}-q_{i, j-1}^{n+1 / 2}}{4 h_{y}}+ \\
+\psi_{y L} v \frac{q_{i, j}^{n+1 / 2}-q_{i, j-1}^{n+1 / 2}}{h_{y}}+\psi_{y R} v \frac{q_{i, j+1}^{n+1 / 2}-q_{i, j}^{n+1 / 2}}{h_{y}}=\frac{3}{2} \mu \frac{q_{i, j+1}^{n+1 / 2}-2 q_{i, j}^{n+1 / 2}+q_{i, j-1}^{n+1 / 2}}{h_{y}^{2}}
\end{gathered}
$$


where $\psi_{x L}=1, \psi_{x R}=0$ for $u>0$ and $\psi_{x L}=0, \psi_{x R}=1$ for $u<0 ; \psi_{y L}=1, \psi_{y R}=0$ for $v>0$ and $\psi_{y L}=0, \psi_{y R}=1$ for $v<0$.

\section{The results of the study and their discussion}

The developed model, the algorithms that implement it, and the constructed software complex allowed to carry out the numerical experiments to simulate the propagation of pollutants in the ground layer of the atmosphere in the presence of forest massifs. The influence of the presence of vegetation on the distribution of pollutants under the action of ascending air currents was studied. Initial data: air density is $1.29 \mathrm{~kg} / \mathrm{m}^{3}$; density of emission is $1.4 \mathrm{~kg} / \mathrm{m}^{3}$; ambient temperature is $200^{\circ} \mathrm{C}$; emission temperature is $1200^{\circ} \mathrm{C}$; speed of air flow is $1 \mathrm{~m} / \mathrm{s}$ in the direction of the coastal area; specific emission power is 5 $1 / \mathrm{s}$; air velocity at the left boundary is $1 \mathrm{~m} / \mathrm{s}$; air permeability coefficient of the vegetative cover is $50 \%$; the height of the vegetation cover is $30 \mathrm{~m}$, the width of the vegetation cover area is $50 \mathrm{~m}$ [5]. The palette shows the concentration of the impurity. Numerical experiments have been performed to simulate the movement of pollutants in the presence of forest plantations (Figure 2) and in the absence of them (Figure 3). The area of forest plantations is indicated by a rectangle.

The color gradation illustrates the concentration of the impurity. Analysis of Figures 3 allows to conclude that the presence of forest plantations influences the distribution of pollutants. Impurities under the influence of ascending air currents rise in the area of forest plantations. Figure 4.a shows the movement of the air in the presence of forest plantations. Gradation of color shows the intensity of the air movement.

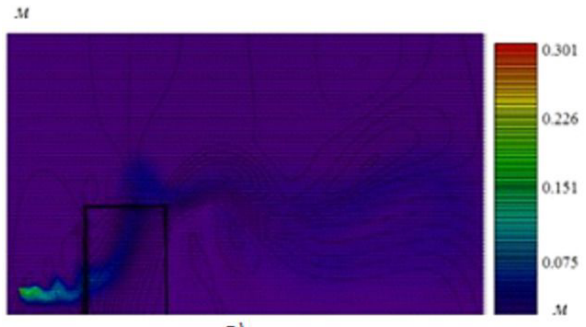

a)

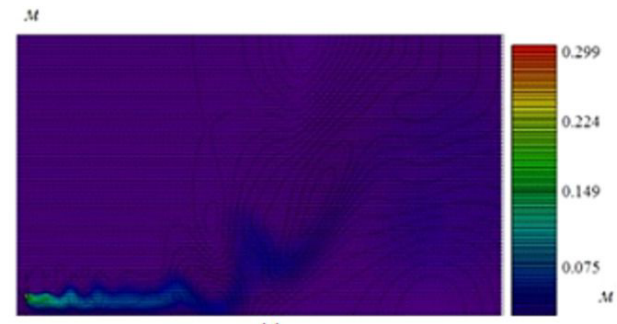

b)

Fig. 3. The field of impurity concentrations taking into account vegetation (a) and without them (b).

The figure illustrates the speed field of the air in the presence of forest plantations and the vortex structures of the air flow located beyond the forest plantations in the direction of the wind movement. The speed of air movement is increased three times in separate areas.

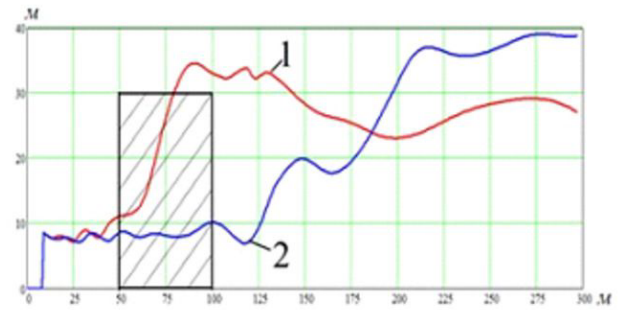

a)

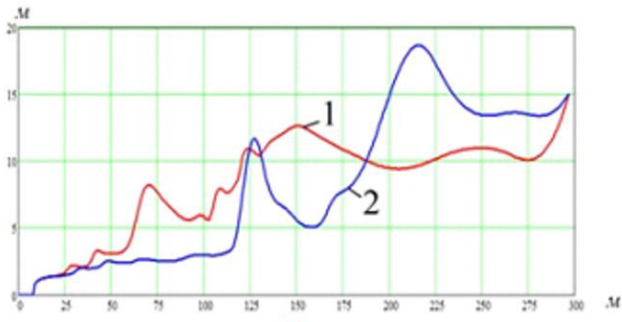

b)

Fig. 4. Trajectories for the distribution of pollutants taking into account vegetation (a) and without them (b). 
Figure 4.a shows the decrease in the width of the current tube in the area of forest plantations. Figure 4.b shows the speed field of the air in the case of forest plantations. A palette shows the intensity of the air movement.

The presence of forest plantations affects the distribution of pollutants. Impurities under the influence of ascending air currents rise in the area of forest plantations. Figure 4.a shows the trajectories of pollutants: 1 - the presence of a semipermeable area, 2 - the absence of a semipermeable area. Figure 4.b shows the dependence of the width of the current tubes, over which the pollutants spread, in the case of a semipermeable region and its absence.

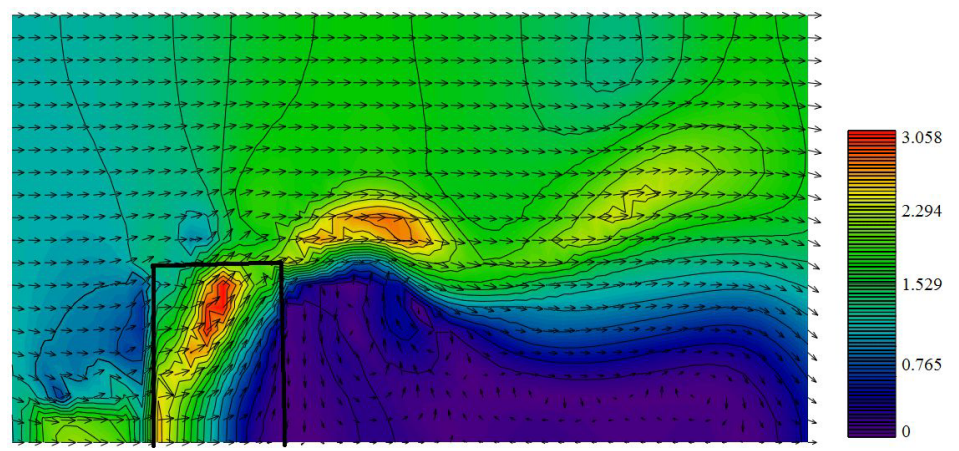

Fig. 5. Movement of the air in the presence of forest plantations.

Figure 5 illustrates the vortex structures of the air flow, located beyond the forest plantations in the direction of wind movement. The speed of air movement is increased three times in separate areas. Next, the influence of the width of the forest plantations area on the air velocity and impurity concentration fields was investigated.

\section{Conclusions}

The proposed methods of mathematical modeling of the motion of air currents enable to evaluate the influence of forest plantations on the propagation of pollutants in the ground layer of the atmosphere and the change in the coefficient of turbulent exchange. The developed model takes into account the transition of water from liquid to gaseous state, precipitation of matter, transport of impurities and heat, heat exchange between liquid and gaseous states.

The scientific novelty of the presented results is that the scheme obtained as a result of a linear combination of the central difference scheme and the "CABARET" scheme was used for the approximation of the convection operator. The use of "CABARET" schemes allows to increase the conditions for the applicability of the developed model. The constructed algorithms are implemented in the form of a software complex that allows to determine the influence of the presence of vegetation on the distribution of pollutants under the influence of ascending air currents. The developed model, the algorithms that implement it, and the constructed software complex allowed the numerical experiments to simulate the propagation of pollutants in the ground layer of the atmosphere in the presence of forest massifs. The advantage of the model of the air environment motion is the possibility of taking into account the influence of forest massifs and turbulent mixing in the equation of continuity of the medium.

This study was supported in part by task no. 2.6905.2017/8.9 within the basic part of the state task of the Ministry of Education and Science 


\section{References}

1. A.I. Sukhinov, A.E. Chistyakov, E.V. Alekseenko, Mathematical Models and Computer Simulations, 3, 5, 562 (2011)

2. A.I. Sukhinov, A.A. Sukhinov, Parallel Computational Fluid Dynamics 2004: Multidisciplinary Applications, 231 (2005)

3. V.S. Vasiliev, A.I. Sukhinov, Matem. Modelling, 15, 10, 17 (2003)

4. I.O. Leontyev, Coastal Dynamics: Waves, Moving Streams, Deposits Drifts (GEOS, Moscow, 2001)

5. E. Alekseenko, B. Roux, A. Sukhinov, R. Kotarba, D. Fougere, Nonlinear Processes in Geophysics, 20, 2, 189 (2013)

6. A.I. Sukhinov, A.E.Chistyakov, E.A. Protsenko, Mathematical Models and Computer Simulations, 6, 4, 351 (2014)

7. G.I. Marchuk, V.I. Agoshkov, Introduction to Projection-grid Methods (Science. The Main Edition of Physics and Mathematics, Moscow, 1981)

8. I.A. Vaseva, A.V. Kofanov, V.D. Liseikin, Y.V. Likhanova, A.M. Kharitonchik Zhurnal Vychislitel'noi Matematiki i Matematicheskoi Fiziki, 50, 1, 99 (2010)

9. A.A. Samarskii, A.V. Gulin, Numerical Methods of Mathematical Physics. 2-nd ed. (The scientific world, Moscow, 2003)

10. O.M. Belotserkovsky, Numerical Modeling at Mechanics Continuous Environments (Fizmatlit, Moscow, 2004)

11. A.I. Sukhinov, A.E. Chistyakov, E.F. Timofeeva, A.V. Shishenya, Mathematical Models and Computer Simulations, 5, 2, 122 (2013)

12. O.M. Belotserkovskii, Turbulence: New Approaches (Nauka, Moscow, 2003).

13. A.A. Samarskii, The Theory of Difference Schemes (Science, Moscow, 1989).

14. O.M. Belotserkovskii, V.A. Gushchin, V.V. Shchennikov, USSR Computational Mathematics and Mathematical Physics, 15, 1, 190 (1975)

15. A.V. Nikitina, A.I. Sukhinov, G.A. Ugolnitsky, A.B. Usov, A.E. Chistyakov, M.V. Puchkin, I.S. Semenov, Mathematical Models and Computer Simulations, 9, 1, 101 (2017)

16. A.I. Sukhinov, A.E. Chistyakov, I.I. Levin, I.S. Semenov, A.V. Nikitina, A.A. Semenyakina, 2016 5th International Conference on Informatics, Electronics and Vision, ICIEV 2016, 1128 (2016)

17. A. Sukhinov, A. Chistyakov, A. Nikitina, A. Semenyakina, I. Korovin, G. Schaefer, 2016 5th International Conference on Informatics, Electronics and Vision, ICIEV 2016, 1134 (2016)

18. A.I. Sukhinov, D.S. Khachunts, A.E. Chistyakov, Computational Mathematics and Mathematical Physics, 55, 7, 1216 (2015)

19. A.I. Sukhinov, A.E. Chistyakov, A.V. Shishenya, Mathematical Models and Computer Simulations, 6, 3, 324 (2014)

20. N. Buzalo, P. Ermachenko, T. Bock, A. Bulgakov, A. Chistyakov, A. Sukhinov, E. Zhmenya, (...), N. Zakharchenko, Procedia Engineering, 85, 84 (2014)

21. A.I. Sukhinov, A.E. Chistyakov, Mathematical Models and Computer Simulations, 4, 4, $398(2012)$ 